\title{
A MODEL FOR TEXTURE DEVELOPMENT DOMINATED BY DEFORMATION TWINNING: APPLICATION TO ZIRCONIUM ALLOYS
}

\author{
C. N. TOME', R. A. LEBENSOHN' ${ }^{1}$ and U. F. KOCKS ${ }^{2}$ \\ 'Instituto de Fisica Rosario (CONICET-UNR), 27 de Febrero 210 bis, 2000 Rosario, Argentina \\ and ${ }^{2}$ Center for Materials Science, MS K765, Los Alamos National Laboratory, \\ Los Alamos, NM 87545, U.S.A.
}

(Received 8 October 1990; in revised form 25 April 1991)

\begin{abstract}
In many polycrystals of less than cubic crystal symmetry, plastic deformation is dominated by twinning. In particular, we will treat the case of $\mathrm{Zr}$ and $\mathrm{Zr}$ alloys in detail. We propose a new method for modelling grain reorientation due to twinning, which is based on a Volume Fraction Transfer (VFT) scheme; the scheme is also applied to the slip modes. We find that this method predicts textures that are, when twinning is the dominant mode, considerably different from, and in better agreement with experiment than the conventional schemes which reorient an entire grain when some criterion has been met. Various combinations of slip and twinning modes and of the associated critical stresses are systematically investigated for the case of rolling, tension and compression of $\mathrm{Zr}$ alloys. A comparison of various predicted and experimental textures leads to the conclusion that twinning must, indeed, be controlling texture development.
\end{abstract}

\begin{abstract}
Réume-Dans de nombreux polycristaux ayant une symétrie inférieure à la symétrie cubique, la déformation plastique est dominée par le maclage. En particulier, nous traitons en détail le cas du zirconium et des alliages de zirconium. Nous proposons une nouvelle méthode pour modéliser la réorientation des grains due au maclage, méthode qui est basée sur le concept de transfert de la fraction volumique (TFV); la méthode est aussi appliquée aux modes de glissement. Nous trouvons que la méthode prévoit des textures qui, lorsque le maclage prédomine, sont considérablement différentes et en meilleur accord avec l'expérience que les schémas traditionnels qui réorientent un grain entier lorsque qu'un certain critère est satisfait. On étudie systématiguement les diverses combinaisons de glissement et de maclage ainsi que les diverses combinaisons des contraintes critiques associées dans le cas du laminage, de la traction et de la compression des alliages de $\mathrm{Zr}$. Une comparaison des diverses textures prévues et expérimentales conduit à la conclusion que la maclage doit, en vérité, contrôler le développement de la texture.
\end{abstract}

\begin{abstract}
Zosammenfasenng - In vielen Polykristallen von Materialien mit weniger als kubischer Symmetrie ist die plastische Verformung geprägt von Zwillingsbildung. Insbesondere behandeln wir hier den Fall des Zirkons und seiner Legierungen ausführlich. Wir schlagen eine neue Methode zur modellhaften Beschreibung der Kornumorientierung durch Zwillingsbildung vor; die Methode beruht auf dem Verfahren der 'Ubertragung des Volumanteils', mit dem auch die Gleitmoden beschrieben werden können. Diese Methode sagt Texturen voraus, die für den Fall, daB Zwillingsbildung vorherrscht, besser mit dem Experiment übereinstimmen und die sich beträchtlich von denen unterscheiden, die mit herkömmlichen Verfahren ermittelt werden, also bei denen ein ganzes Korn umorientiert wird, wenn ein bestimmtes Kriterium erfüllt ist. Für die Fälle des Walzens, der Zug- und der Druckverformung von Zirkonlegierungen werden verschiedene Kombinationen von Gleit- und $Z$ willingsmoden und die zugehörigen kritischen Spannungen systematisch untersucht. Aus einem Vergleich verschiedener vorausgesagter und experimenteller Texturen folgt, daß Zwillingsbildung tatsächlich die Entwicklung der Textur steuert.
\end{abstract}

\section{INTRODUCTION}

Close-packed hexagonal metals are the non-cubic materials for which polycrystal plasticity has been most closely investigated. Besides their intrinsic plastic anisotropy, they exhibit the two complicating characteristics common to all non-cubics: twinning may be a major deformation mode and the total number of slip and twinning modes may be large. In this case the Critical Resolved Shear Stresses (CRSS) for all modes are often not known a priori and, moreover, they may strongly depend on temperature, alloy content, grain size, etc. It is never- theless possible to make rather specific predictions about possible deformation textures if one takes advantage of the fact that there are domains of CRSS ratios over which texture development is insensitive to the precise values within these domains. This occurs whenever the topology of the Single Crystal Yield Surface (SCYS) is not changed by a change in the CRSS's.

In the next Section we will first review the importance of the SCYS for polycrystal deformation and show, in particular, which modes can accommodate which components of the prescribed strain-rate tensor. We will emphasize the cases where twinning 
is used not only to provide strains in the uniaxial extension or compression of the $c$-axis, but also to accommodate shears in the basal plane. We will show that in the latter case twinning dominates the deformation.

Twinning itself is a more complicated deformation mode than slip because it not only contributes to the incremental reorientation of the grain just as slip does, but also produces a volume fraction of the grain with a drastically different orientation than the matrix: in a certain sense, it produces new grains. Since a computer simulation cannot allow any signifcant persistent increase in the number of grains, various algorithms have been invented to represent this discrete reorientation concisely. In Section 3 we will first review briefly previous treatments of twinning in texture development codes and then we will introduce two new ones: the "Predominant Twin Reorientation" (PTR) and the "Volume Fraction Transfer" (VFT) schemes. The latter, in particular, proves to be successful in treating deformation texture development in $\mathrm{Zr}$.

After describing details of the calculation in Section 4, we present textures for rolling and uniaxial tensile and compressive straining for various combinations of deformation systems. We will conclude that any significant contribution from $\langle c+a\rangle$ slip is in contradiction to the observed deformation textures in hexagonal metals and that, conversely, dominant tensile and compressive twinning yields the best agreement with observed texture in $\mathrm{Zr}$ alloys.

\section{THE SCYS FOR Zr ALLOYS}

In a previous work, Tomé and Kocks [1] propose a systematics for grouping the vertices of the SCYS and for characterizing its topology according with the crystal symmetry and the CRSS on the slip and twinning systems. Though we do not make explicit use of the vertices of the SCYS in the present calculations, we will use this framework to discuss texture evolution as related to the topology of the SCYS and the active deformation modes.

In what follows we will represent deviatoric stress and plastic strain-rate tensors in terms of 5 independent components using the convention proposed by Lequeu et al. [2] (with the first two components interchanged)

$$
\begin{aligned}
& \bar{\sigma}=\sqrt{ } 2\left(\sqrt{ } 3 \frac{\sigma_{33}^{\prime}}{2}, \frac{\sigma_{22}-\sigma_{11}}{2}, \sigma_{23}, \sigma_{13}, \sigma_{12}\right) \\
& \dot{\bar{\epsilon}}=\sqrt{ } 2\left(\sqrt{ } 3 \frac{\dot{\epsilon}_{33}}{2}, \frac{\dot{\epsilon}_{22}-\dot{\epsilon}_{11}}{2}, \dot{\epsilon}_{23}, \dot{\epsilon}_{13}, \dot{\epsilon}_{12}\right) .
\end{aligned}
$$

According with this convention the Schmid law becomes

$$
m_{\mathrm{k}}^{\mathrm{s}} \sigma_{\mathrm{k}}=\tau^{\mathrm{s}}
$$

where $\tau^{s}$ is the CRSS in the system $s$ and $m_{\mathrm{k}}$ are the vector components of the tensor $m_{i j}=\frac{1}{2}\left(n_{i} b_{j}+b_{i} n_{j}\right)$, defined with the same convention of equations (1) and (2). The plastic deformation associated with the shear strain-rate $\dot{\gamma}^{\mathrm{s}}$ in system $\mathrm{s}$ is given by the kinematic equation

$$
m_{\mathbf{k}}^{\mathrm{s}} \dot{\gamma}^{\mathrm{s}}=\dot{\epsilon}_{\mathbf{k}}
$$

It is evident from equation (4) that a system cannot produce a deformation component $\epsilon_{\mathrm{k}}$ unless the corresponding component $m_{k}$ is non-zero.

Equation (3) associates a plane in 5 dimensional stress space to every deformation system, the distance of that plane to the origin being proportional to the CRSS of the system $\tau$. The SCYS is a faceted polyhedron in stress space, defined by the inner envelope of those planes and a "vertex" is defined by the intersection of at least 5 facets. Chin and Mammel [3] first realized that a system is never active if the associated plane is beyond the inner envelope defined by the SCYS and identified intervals of CRSS's within which certain deformation modes are active. Tomé and Kocks [1] show that those domains can be partitioned into subdomains of CRSS's where the configuration of the vertices, and as a consequence the associated cone of normals, remain invariant. A property of those "topological domains" of the SCYS is that for any combination of CRSS's within the domain exactly the same system activity and texture evolution is predicted when the Taylor-Bishop-Hill approach is used. Tomé and Kocks analyze those domains for the case of hexagonal lattices deforming by $\langle a\rangle$ prism slip, $\langle c+a\rangle$ pyramidal slip and tensile twins. Takeshita et al. [4] carry out a similar analysis for the case of trigonal lattices and calculate texture development in calcite. They observe that only few transitions between topological domains are accompanied by significant changes in the resulting texture. The same is true for hexagonal materials and in what follows we identify, for the systems characteristic of $\mathrm{Zr}$ alloys, the main domains of CRSS's which lead to qualitatively different textures. The case of titanium and $\mathrm{Ti}$ alloys, which present basal slip and less twinning activity, is analyzed in a coming paper [5].

The potentially active deformation modes to be considered here are those reported by Tenckhoff [6] for zirconium alloys at room temperature: $\{10 T 0\}\langle 1210\rangle$ prism slip; pyramidal slip in the $\{10 \Gamma 1\}$ plane, both in the $\langle 1123\rangle$ and $\langle 1210\rangle$ directions; $\{10\lceil 2\}\langle 10\lceil 1\rangle$ tensile twins and $\{21\lceil 2\}\langle 21\lceil 3\rangle$ compressive twins. Examination by TEM of $\mathrm{Zr}$ and Zircaloy samples done by Philippe et al. $[7,8]$ supports the evidence for cross slip in $\{10 \Pi 1\}$ planes in the $\langle 1210\rangle$ direction. The following convention will be used in this work when referring to the deformation modes: $\operatorname{pr}\langle a\rangle, \operatorname{pyr}\langle c+a\rangle$ and $\operatorname{pyr}\langle a\rangle$ for the slip systems and ttw, ctw for the tensile and compressive twins respectively. Because of its low CRSS, prismatic slip is always observed in zirconium alloys, but it alone does not "close" the SCYS and 
other modes are required even if their CRSS's are much higher than $\tau^{\text {pr }\langle a\rangle}$.

It is convenient to consider 3 subspaces of the 5 -dimensional space. First, if the $c$-axis is labeled 3, the first strain component in equation $(2)\left(\epsilon_{1}=\epsilon_{33}\right)$ is associated with uniaxial deformation in the $c$-axis direction. It is well known that among all the slip modes only $\langle c+a\rangle$ pyramidal slip has non-zero $m_{1}$ components in equation (4) and can accommodate this deformation; also the twinning systems provide this deformation component but only either the positive (extension) or negative (compression) deformation. Thus, so long as any model close to Taylor's is used, either $\langle c+a\rangle$ is needed or one twinning mode in connection with $\langle c+a\rangle$, or two twinning modes, one tensile and one compressive.

Second, we consider the subspace in which prism slip provides deformation modes. This space consists of $\left(\epsilon_{22}-\epsilon_{11}\right) / 2$ and $\epsilon_{12}$ : two shears of the basal plane at $45^{\circ}$ with respect to each other. In the vector nomenclature of equation (2) these are the components $\epsilon_{2}$ and $\epsilon_{5}$ respectively. Prism slip is observed to be a prominent deformation mode in all hep metals with the exception of $\mathrm{Zn}$ and $\mathrm{Cd}$; we will assume it to be so here.

Finally, there are two dimensions left that could be satisfied by basal slip if it were present: the subspace formed by the shears $\epsilon_{23}\left(\epsilon_{3}\right)$ and $\epsilon_{13}\left(\epsilon_{4}\right)$. In this paper we are concerned only with polycrystal deformation in the absence of basal glide (as appropriate for $\mathrm{Zr}$ at low and intermediate temperatures). The question then is whether deformation in these two dimensions is accommodated by $\langle c+a\rangle$ slip or by twinning. In either case, mixtures of the modes that give $c$-axis extension with those that give $c$-axis compression are required. Another slip mode that could help close this subspace of the SCYS is pyramidal slip in the $\langle a\rangle$ direction. Although pyr $\langle a\rangle$ does not provide deformation along the $c$-axis, it provides shear components in the other four dimensions and, as a consequence, reduces the amount of twinning and $\langle c+a\rangle$ slip required to accommodate deformation.

To consider the situation quantitatively we will first assume that pyr $\langle a\rangle$ is absent as well as basal glide, and that prismatic slip is easy compared to $\langle c+a\rangle$ slip and twinning (for numerical purposes $\tau^{\operatorname{pr}\langle a\rangle}$ is taken, in what follows, as half the minimum CRSS of the other systems). The resulting topological map for various ratios of CRSS's of tensile and compressive twins with respect to $\langle c+a\rangle$ slip is shown in Fig. 1(a). This map identifies four domains of CRSS's with the following characteristics: in addition to prism slip, only $\langle c+a\rangle$ slip is active in the domain 3 , defined by $\tau^{\text {ttw }}>1.23 \tau^{\text {pyr }\langle c+a\rangle}$ and $\tau^{\mathrm{ctw}}>1.11 \tau^{\mathrm{pyr}\langle c+a\rangle} ;\langle c+a\rangle$ slip and tensile twins are active in 4 ; some contribution of all three modes is present in domain 5; and only tensile and compressive twins are active in 6 . The domains are numbered in correspondence to the figures in Section 4, where the different combinations of deformation modes are explored.

The topological map of Fig. I(b) incorporates pyramidal $\langle a\rangle$ in addition to the previous systems. As before, no attempt is made at plotting $\operatorname{pr}\langle a\rangle$ because it is always present in the SCYS. In order to incorporate another system into the map, tensile and compressive twins are assumed to have the same CRSS ( $\left.\tau^{\mathrm{tw}}=\tau^{\mathrm{ctw}}=\tau^{\mathrm{tw}}\right)$ and are plotted in the same axis. Figure 1(b) shows that when $\tau^{\text {pyr }\langle c+a\rangle}<0.86 \tau^{\text {pyr }\langle a\rangle}$ or $\tau^{i w}<0.42 \tau^{\text {pyr }\langle a\rangle}$, the $\operatorname{pyr}\langle a\rangle$ systems are outside the SCYS, in which case we fall into one of the four topological domains described in Fig. 1(a). In the interval $0.86<\tau^{\mathrm{pyr}\langle c+a\rangle} / \tau^{\mathrm{pyr}\langle a\rangle}<1.25$, $\operatorname{pyr}\langle a\rangle$ is part of the SCYS but $\operatorname{pyr}\langle c+a\rangle$ and twinning systems prevail and the associated textures are not changed significantly by the low activity of the pyr $\langle a\rangle$ systems. Only for $\tau^{\text {pyr }\langle c+a\rangle} / \tau^{\operatorname{pyr}\langle a\rangle}>1.25$

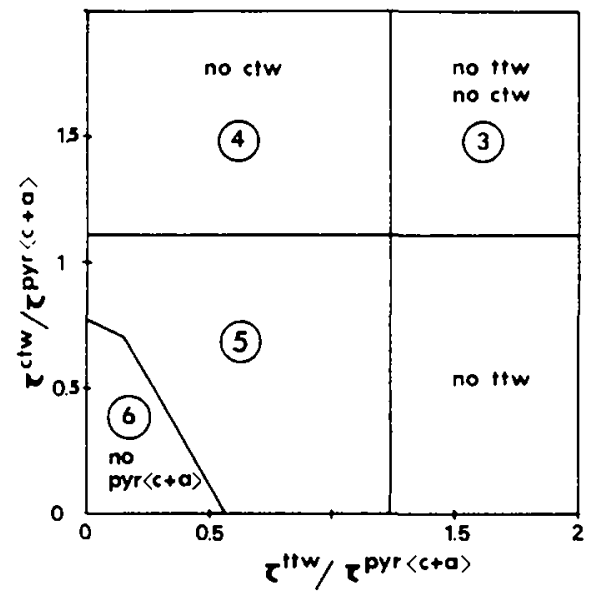

(a)

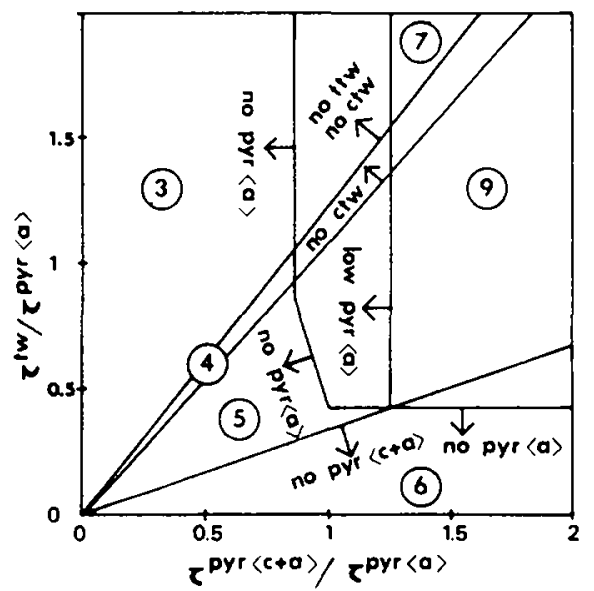

(b)

Fig. 1. Main topological domains of the Single Crystal Yield Surface for hexagonal systems defining the combinations of CRSS's that lead to similar textures. In all cases $\tau^{\text {pr }\langle a\rangle}$ is taken as half the minimum CRSS of the remaining deformation modes. 
the inclusion of pyr $\langle a\rangle$ makes a difference in the resulting textures, either in combination with twinning and pyr $\langle c+a\rangle$ (domain 9) or in combination with $\operatorname{pyr}\langle c+a\rangle$ alone (domain 7 ).

\section{TWINNING MODELS}

In this Section we discuss the treatment of twinning when modelling deformation textures. A brief review of previous work in that respect will provide the necessary perspective on the subject.

Chin and Mammel [9] were the first to incorporate twinning within the maximum work formulation of Bishop and Hill [10] by assuming that twins are activated by a directional CRSS in the twinning plane. Those authors were also the first to realize that there is a competition among different deformation modes and that some modes become inoperative when the associated CRSS exceeds certain values [3]. Thornburg and Piehler [11] calculate the reorientation tendencies-but not texture developmentassociated with rolling of titanium alloys. These authors assume that the deformation is accommodated by $\langle a\rangle$-slip in prism, pyramidal and basal planes and, in order to provide deformation along the $c$-axis, also by tensile and compressive twins. No attempt is made at analyzing the dependence of reorientation with the CRSS in those systems. In a latter paper Thornburg and Piehler [12] improve the treatment of Chin and Mammel [13] and provide a systematic analysis of the vertices of the SCYS in terms of the relative values of the CRSS's. In both works (Refs [11] and [12]) only the vertices with at least four slip systems associated are considered.

To our knowledge, Van Houtte [13] is the first to propose a way of dealing with reorientation by twinning during the simulation of texture development and to apply the model for the prediction of rolling textures in f.c.c. brass, assuming $\{111\}\langle 110\rangle$ slip and $\{111\}\langle 112\rangle$ twinning. Without further modification the Van Houtte's scheme has been applied to calculate texture development in hexagonal materials with deformation modes typical of titanium alloys $[14,15]$ and also in materials of geological interest like calcite, which deforms predominantly by twinning [4]. We will review Van Houtte's scheme in this Section and improve upon it in the following.

Typically, when simulating texture development due to plastic deformation, one represents the polycrystal using a finite set of (perhaps weighted) orientations in Euler space, each one representing a volume fraction $f^{n}$. The total deformation of the polycrystal is achieved by imposing successive strain increments to those grains and calculating the necessary shears in the deformation systems. The final texture is given by the grain reorientations associated with those shears. When only slip systems are active one can reasonably assume that each grain deforms homogeneously and that it reorients gradually as a whole.
The activation of twinning systems changes this picture in two fundamental aspects: firstly, instead of being homogeneous, deformation localizes in a volume fraction of the grain $\Delta g^{n t_{1}}=\Delta \gamma^{n, t_{1}} / S$, where $\Delta \gamma^{n . t_{i}}$ is the shear strain contributed by the twinning system $t_{t}$ in the grain $n$, and $S$ is the characteristic shear of the twin $(S=0.167$ for ttw and $S=0.225$ for ctw). This grain fraction represents a polycrystal volume fraction

$$
\Delta f^{n, s_{i}}=f^{n} \frac{\Delta \gamma^{n, s_{i}}}{S}=f^{n} \Delta g^{n, \ell_{i}}
$$

Secondly, the twinned fraction adopts a characteristic orientation with respect to the initial matrix, which for the purpose of the simulation amounts to splitting the original orientation into two grains, preserving the original volume fraction. Leaving aside the question of how the twinned part and the matrix interact from then on and assuming that they can be treated independently of each other, the problem that remains is that the number of orientations represented by the twinned fractions increases geometrically with the simulation, overflowing quite rapidly the processing capabilities of the computer.

\subsection{Van Houtte's model}

The approach proposed by Van Houtte [13] for avoiding the problems associated with modelling reorientation by twinning relies on statistical considerations and does not increase the number of crystallite orientations. Instead, after a deformation step each grain is either reoriented by slip or it is given one of the twinning related orientations. The criterion for making the decisions is based on the relative volume fractions of the non-twinned and the twinned parts: the reorientation by slip is assigned an a priori probability $\left(1-\Delta g^{n, t_{1}}\right)$ and the reorientation by twinning a probability $\Delta \boldsymbol{g}^{n, t_{i}}$. All twinning systems are scanned and the grain is completely reoriented by twinning if the outcome of a random number generator falls in the interval 0 to $\Delta g^{n, t_{1}}$. For being statistically meaningful, this approach requires that several grains in the collection must have the same or very similar orientations if the proportion between twinned and untwinned material given by this MonteCarlo approach is to be close to the "exact" value $\Delta g^{n, t_{i}}$. Considering that a partition of the reduced Euler space in cells of 10 degrees on a side leads to a total of about 2000 points in orientation space, it is numerically not feasible to have each orientation represented many times. Another drawback of the method is that the decision for reorienting the grain is based on the volume fractions calculated at every incremental step and is independent of the previous deformation history in the grain. As a consequence, it may occur that the selected twinning system is not among the more active ones throughout deformation. Once again, this objection would not apply when dealing with a statistically meaningful number of orientations. 


\section{2. "Predominant Twin Reorientation" scheme}

Our first approach resembles Van Houtte's proposal in that we also pick as the new orientation either the one of the untwinned part or the orientation associated with one of the active twinning systems. However, it differs from Van Houtte's in two important aspects: (i) a different criterion is used for making the choice; (ii) the accumulated volume fraction represented by the twin-reoriented grains is made to match the accumulated twinned fraction associated with the twinning shears $\Delta \gamma^{n, t_{1}}$ given by equation (5).

The procedure is as follows. In each grain the imposed deformation is allowed to be accommodated by slip and twinning and the fraction of the grain $\Delta g^{n, t_{i}}$ associated with each twinning system $t_{i}$ is accumulated as deformation proceeds

$$
g^{n, t_{t}}=\sum_{\text {steps }} \Delta g^{n, t_{s}}
$$

Adding up over all systems and over all grains we obtain the "real" twinned fraction in the polycrystal

$$
F_{\mathrm{R}}=\sum_{m} f^{n} \sum_{i_{t}} g^{n, t_{t}}
$$

which is the magnitude accessible to experimental measurement. At the same time, whenever a grain is completely reoriented by twinning the volume fraction $f^{n}$ that it represents is accumulated into an "effective" polycrystal twinned fraction

$$
F_{\mathrm{E}}=\sum_{n} f^{n}
$$

where the sum is performed over the reoriented grains only. At every incremental step the fraction accumulated in the individual twinning systems of each grain $\boldsymbol{g}^{n, t_{1}}$ is compared against a "threshold" fraction $F_{\mathrm{T}}$ defined in terms of polycrystal parameters by the empirical algorithm

$$
F_{\mathrm{T}}=0.25+0.25 \frac{F_{\mathrm{E}}}{F_{\mathrm{R}}} .
$$

If $g^{n, t_{1}}$ is larger than $F_{\mathrm{T}}$ the grain is completely reoriented according to that twinning system. Two things are achieved with this procedure: firstly, reorientation by twinning takes place in those sysems which exhibit highest activity in the grain throughout the deformation process instead of being decided randomly at each strain increment. This principle is meant to be reflected in the designation "Predominant Twin Reorientation". Secondly, as more grains are reoriented by twinning $F_{\mathrm{E}}$ growths larger than the accumulated fraction $F_{\mathrm{R}}$, the threshold fraction $F_{\mathrm{T}}$ increases, and further reorientation by twinning is inhibited until the accumulated fraction $F_{\mathrm{R}}$ "catches up" as deformation proceeds. Such procedure is self-controlling and both fractions, $F_{\mathrm{E}}$ and $F_{\mathrm{R}}$, tend to remain approximately equal throughout deformation. The coefficients which appear in equation (9) were arrived at as follows: the effect of the constant term is to delay the reorientation by twinning until the grain exhibits a defined trend and at least $25 \%$ of it has twinned. Once the grains start reorienting $F_{\mathrm{E}}$ increases rapidly and the second term fluctuates around the value 0.25 after a few incremental steps. As a consequence of equation (9), when about $50 \%$ of the grain's volume has twinned on a given system the grain orientation is replaced by the one of the twin. It is important to note that the criterion is based on the volume fractions and not on the shears associated with twinning because it is the former which are relevant to texture.

A disadvantage of the PTR method is, besides imposing the reorientation of the whole grain, that it only accounts for the most active twinning system in each grain. We expect it to be correct when one twinning system prevails upon the others but may not be realistic when several twinning systems make similar contributions to deformation within a given grain. In hexagonals it is not uncommon to observe more than one primary active twins per grain. Besides, even if multiple twinning activity is not observed in individual grains, it is to be expected that local fuctuations inside the polycrystal will favour different twinning systems in grains having similar orientations but different neighbourhoods. Since each grain used to simulate the polycrystal is representative of all grains with similar orientations, the method employed has to reflect such "average" characteristic and permit multiple twinning. This consideration underlies the "Volume Fraction Transfer" scheme that we present in the following section.

\section{3. "Volume Fraction Transfer" scheme}

The purpose of this method is to account for all twinning reorientations and their associated volume fractions. The polycrystal is represented, as before, by means of a set of discrete orientations with proper weights. However, it differs from the classical methods for simulating texture development in that the orientations are kept fixed while the associated volume fractions are allowed to evolve during deformation.

To start with, the Euler space is divided into identical and approximately equiaxed cells of volume $\left(\Delta \phi_{1} \times \Delta \theta \times \Delta \phi_{2}\right)$, each cell containing an initial volume fraction $f^{n}$ and representing an orientation defined by the coordinates of its center: $\phi_{1}^{n}, \theta^{n}, \phi_{2}^{n}$ (Bunge's convention is assumed in this work for defining the Euler angles). Assume, to start with, that only the slip systems are active and impose a small strain increment to the grain. The reorientation that this grain will experience can be represented as a displacement vector $\left(\delta \phi_{1}, \delta \theta, \delta \theta_{2}\right)$ in Euler space and, if the dimensions of the cell are small, one may assume that every point within the cell will shift by about the same amount, which results in a rigid displacement of the cell as a whole. In this process a portion of the cell overlaps with the neighboring cells. The basis of the scheme is to assume that the volume fraction of material $\Delta f^{\mathrm{nm}}$ contained in the overlapped 
portions is "transferred" from the cell $n$ to the neighboring cell $m$ as a consequence of the strain increment. If the material is assumed to be homogeneously distributed within the cell, then $\Delta f^{n m}$ will be simply proportional to the overlapping volume. Conservation requires that the fractions added to neighboring cells are substracted from cell $n$. This process of transference, repeated after every strain increment, leads to a gradual variation of the volume fraction associated with each cell and so to texture development. The situation is sketched in Fig. 2(a) for a 2-dimensional Euler space, although for numerical purposes the procedure has to be implemented in the 3-dimensional space.

Next, assume that also twinning is active within the grains, in which case we have a certain volume fraction $\Delta f^{n, i l}=f^{n} \Delta \gamma^{n, 1} / S$ associated with the twinning shears [equation (5)]. The orientation of the twinned fraction is crystallographically related to, but completely different from, the grain orientation. As a consequence, a finite and not an incremental displacement takes place in Euler space and the fraction $\Delta f^{n, t_{1}}$ has to be transferred from the cell $n$ to a noncontiguous cell $m$ of the grid. After the twinned fraction is transferred to the cell it is treated independently of the parent grain. The situation is sketched in Fig. 2(b).

An advantage of the VFT scheme described above is that it permits to account exactly for the twinned volume fractions without having to increase the initial number of orientations (although this number must be fairly large). This is achieved by assigning those fractions to an existing orientation instead of keeping track of each one separately. In doing so, the deformation history of the reoriented fraction is lost: the grid orientation has to be regarded as an average representative of all grains having similar orientations though different histories.
When twinning is not active, the VFT scheme should give the same results as the classical scheme; it has, however, the advantage of being computationally faster when the strain increment imposed on each grain is the same throughout the deformation: since the orientation of each grid element is constant, the reorientation is then the same at each step. The volume fraction $f^{n}$ will evolve with deformation but the proportion of it which is transferred to the neighboring cells remains the same and has to be calculated only for the first step. Needless to say, the fact that the reorientations have to be evaluated only for the first step substantially speeds up the calculation. The Volume Fraction Transfer scheme can also be used in combination with self-consistent models of polycrystal plasticity or nonproportional strain paths, though in these cases the incremental deformation of each grain is not constant and the transfered fractions have to be recalculated at every step.

A word is in order at this point concerning previous work along similar lines. Three papers were presented at the 8th International Conference on Textures of Materials (ICOTOM) held in 1987 dealing with an "Eulerian" notion of texture evolution. One was a paper by Tomé et al. [16] in which a 2-dimensional VFT scheme was implemented for performing a self-consistent visco-plastic calculation of texture development during cold rolling. In this case only the twinned fractions were treated according to the VFT approach while the remaining fraction of the grain was treated in the classical way. A similar scheme was proposed by Steinkopff and Matthies [17] for treating the texture associated with martensitic transformation in much the same way as we treat the texture development associated with twinning reorientation. The authors suggest that the scheme can be used for simulations of deformation textures, except

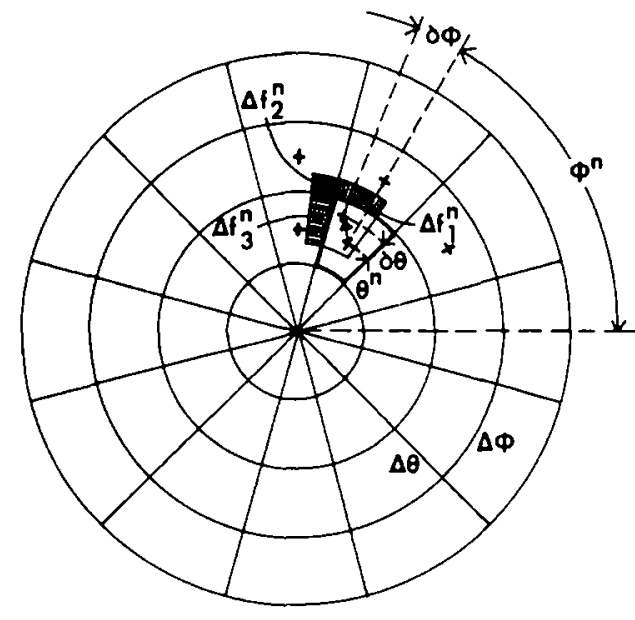

(a)

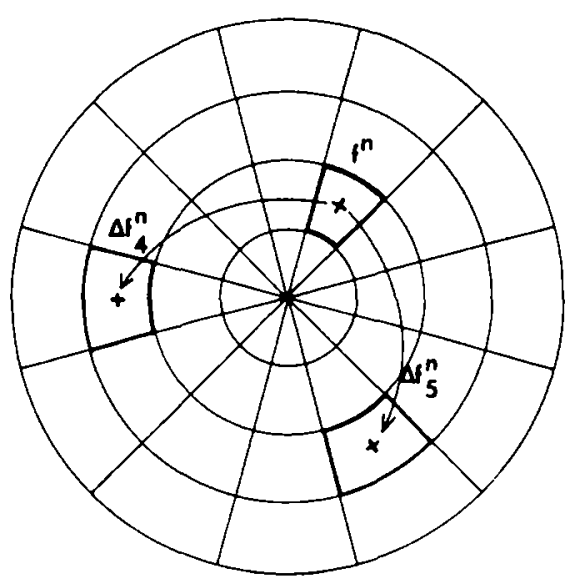

(b)

Fig. 2. Schematic representation of 2-dimensional cells in Euler space showing the volume fractions transferred from cell $n$ : (a) to the neighboring cells due to reorientation by slip; (b) to non-neighboring cells due to twinning reorientation. 
that instead of the concept of cell overlapping they envisage smaller cells and a complete transfer from one cell to another during a typical reorientation step. Two other papers by Esling et al. [18] and Klein et al. [19] approach the subject from a "continuum" point of view. The authors propose to describe texture development by means of a "rotation field" defined in Euler space and derive a set of complicated differential equations that describe the evolution of the coefficients of the Orientation Distribution Function as a function of deformation. In its present formulation the method accounts neither for discontinuities in reorientation, such as the ones associated with twinning activity, nor for changes of the reorientation tendencies with deformation, as a result from self consistent modeling of texture development or nonproportional strain paths.

\section{PREDICTED TEXTURES AND DISCUSSION}

\subsection{Details of the calculation}

The two methods described in Section 3 were implemented for the calculation of texture development, primarily during cold rolling of hexagonal materials starting from a random polycrystal. Within the VFT scheme we take advantage of the hexagonal symmetry of the crystals and we reduce the extent of the Euler space necessary for describing the texture to the following intervals: $0 \leqslant \phi_{1} \leqslant 2 \pi, 0 \leqslant 0 \leqslant \pi / 2$, $0 \leqslant \phi_{2} \leqslant \pi / 3$. The reduced space was partitioned in cells of 10 degrees, which gives a total of 1944 orientations to represent the texture. The initial volume fraction associated with each orientation was chosen such as to give the same density in every cell but it could, instead of being uniform, be chosen to correspond to a measured initial texture. If desired, it is possible to achieve a further reduction of the Euler space by explicitly imposing the orthotropic symmetry of the rolling texture. For the calculations done using the PTR scheme an initial set of 500 random orientations was employed.

In both cases the rate sensitive approach was favored over the classical Taylor-Bishop-Hill criterion for the selection of the slip and twinning systems. Within the rate sensitive formulation the strain rate in every system is given by the constitutive equation

$$
\dot{\gamma}=\dot{\gamma}_{0}\left(\tau_{r}^{s} / \tau_{c}^{s}\right)^{n}
$$

where $\tau_{r}^{s}=m_{i}^{s} \sigma_{i}$ is the resolved shear stress in the system $s, \tau_{c}^{s}$ is a scaling value which we identify with the critical shear stress (at the standard rate $\dot{\gamma}_{0}$ ) and $n$ is the inverse of the rate sensitivity. In this work $n$ was taken equal to 33 , which correspond to a rate insensitive material and guarantees that the contribution of a given system to deformation is negligible unless $\tau_{\mathrm{r}}$ is very close to $\tau_{\mathrm{c}}$. In this limit, equation (10) leads to the same system activity as the Schmid law [equation (3)] and the conclusions derived in Section 2 concerning distinctly defined topological domains of CRSS's hold in full. In the case of rate sensitive materials (say, $n<10$ ) the concept of a SCYS becomes more ambiguous and the predicted textures turn out to exhibit less sharp components. A smooth (as opposed to a sudden) transition is then observed in the predicted textures when crossing from one topological domain to another; still, fairly distinctive textures are associated with each domain of CRSS's. The nonlinear kinematic equation relating the 5 plastic strain-rate components in the grain to the 5 deviatoric stress components is, from equation (4)

$$
\dot{\epsilon}_{k}=\dot{\gamma}_{0} \sum_{\mathrm{s}} m_{k}^{\mathrm{s}}\left(m_{j}^{\mathrm{s}} \sigma_{j} / \tau_{\mathrm{c}}^{\mathrm{s}}\right)^{n}
$$

and can easily be solved by means of a NewtonRaphson technique both for a Taylor or a Relaxed Constraint approximation. The advantage of such an approach is twofold: firstly, since in principle all systems are allowed to be active instead of the 5 required when the Schmid yield criterion is used, no ambiguity in the slip system selection results. A second advantage, shared by linear programming techniques, is that by becoming independent of the maximum work principle there is no need to know in advance the configuration of vertices of the Single Crystal Yield Surface (SCYS), which for hexagonal materials is complicated and does not remain invariant through deformation unless the ratio of CRSS's remains invariant [11].

In this work we present results of texture calculations done using a pure Taylor model. The use of the Relaxed Constraints assumption does not lead to improved textures for the cases considered here and the corresponding results are not presented. The potentially active deformation modes considered are those reported in Section 2, characteristic of zirconium alloys at room temperature: $\{10 \mathrm{~T} 0\}\langle 1210\rangle$ prism slip; pyramidal slip in the $\{10 \overline{1} 1\}$ plane. both in the $\langle 11 \overline{2} 3\rangle$ and $\langle 1510\rangle$ directions; $\{10 \overline{2} 2\}\langle 10 T 1\rangle$ tensile twins and $\{2\lceil T 2\}\langle 2 T\lceil\overline{3}\rangle$ compressive twins. The same convention of Section 2 will be used in this Section when referring to the deformation modes: $\operatorname{pr}\langle a\rangle, \operatorname{pyr}\langle c+a\rangle$ and $\operatorname{pyr}\langle a\rangle$ for the slip systems and $t t w$, ctw for the tensile and compressive twins respectively. All relevant combinations of these modes, each associated with a different topological domain of the SCYS, are explored.

Calculations of rolling, axisymmetric tension and axisymmetric compression textures were performed using the Predominant Twinning Reorientation (PTR) and the Volume Fraction Transfer (VFT) schemes described in Section 3 and using 500 and 1944 orientations respectively. The PTR scheme was implemented in LApp (Los Alamos polycrystal plasticity), a general purpose code for the calculation of texture development, yield surfaces and mechanical properties of textured materials [20]. The VFT scheme was implemented separately [21], although it is also possible to makc it part of an existing code 
and apply the scheme only to the fraction of material that reorients by twinning while treating the remaining fraction with the classical approach, as was done by Tomé et al. [16]. As mentioned previously, the VFT scheme performs faster than the classical one because grain reorientation has to be calculated only for the first incremental step. For the same number of initial orientations CPU times differ by a factor roughly equal to the number of deformation increments imposed to the polycrystal (20 in our case).

Besides the final predicted texture, we find it useful to analyze the relative contributions to deformation of the different modes as a function of strain. We characterize the mode activity in each strain increment by the fraction:

$$
\text { mode fraction }=\frac{\sum_{n} f^{n} \sum_{s(\text { mode })} \Delta \gamma^{n, s}}{\sum_{n} f^{n} \sum_{s \text { (all modes })} \Delta \gamma^{n s s}}
$$

where the numerator is the total plastic shear contributed in each grain by the systems of the mode, summed over all grains and weighted by the grain's volume fraction. The denominator is the plastic shear contributed by all the systems in the grain, summed over all the grains.

\subsection{Rolling textures}

Deformation by rolling was simulated up to $50 \%$ true strain, which corresponds to $40 \%$ thickness reduction. Within the range of CRSS's defined by domain 3 of Fig. 1(a) deformation is accommodated through $\langle a\rangle$ prismatic and $\langle c+a\rangle$ pyramidal slip and texture development leads to a concentration of basal poles along the rolling direction (see Fig. 3). This RD component, which is not observed experimentally, is due only to the activity of the $\langle c+a\rangle$ systems, since prismatic glide does not reorient the $c$-axis. This texture, which is invariant for values of $\tau^{\mathrm{pyr}\langle e+a\rangle}>2 \tau^{\mathrm{pr}\langle a\rangle}$, is the same calculated with the VFT or the classical scheme.

Next, assuming $\tau^{\mathrm{pr}\langle o\rangle}=1, \tau^{\mathrm{pyr}\langle c+a\rangle}=4, \tau^{\mathrm{ttw}}=2$ shifts the CRSS's to the domain 4 of Fig. 1(a) and introduces tensile twins activity which tends to

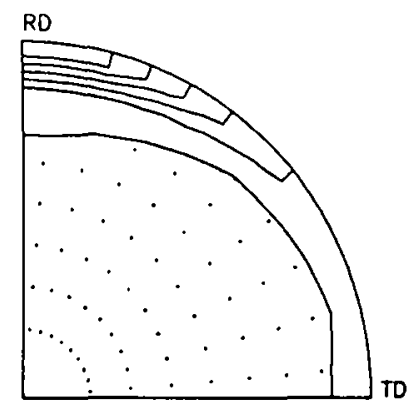

Fig. 3. Case $t^{p r\langle a\rangle}=1, t^{p r\langle c t a\rangle}=4$. Basal pole figure after $40 \%$ thickness reduction in rolling ( 0.5 true strain), obtained with the VFT scheme under FC conditions. The lines correspond to multiples of random orientations and the dots indicate density values lower than 1. Equal area and not stereographic projection is used.

deplete the RD component and give a component in the ND. The final texture is shown in Fig. 4(a) and the relative contribution of the systems, given by equation (12), is shown in Fig. 4(b). Observe that although $\langle c+a\rangle$ slip dominates and twinning shears are small, the latter are very effective in building the ND component because of the high volume fraction associated with tensile twins $\left[S^{\text {ttw }}=0.167\right.$ in equation (5)].

The incorporation of compressive twins with the same CRSS as the tensile twins $\left(\tau^{\mathrm{ctw}}=2\right)$ shifts the calculation to the topologic domain 5 in Fig. (1a). The effect on the basal pole figure is to tilt the normal component by about $20^{\circ}$ towards the TD and also to give a component in the TD [see Fig. 5(a)] but without modifying the RD component. It is interesting at this point to compare the predictions of the VFT and the PTR schemes: as can be seen in Fig. 5(c) the PTR scheme predicts neither the tilted nor the TD component. Since the evidence of Fig. 5(b) and (d) indicates that the system activity is similar for both simulations, we attribute the discrepancy to the criterion used for deciding grain reorientation. As a matter of fact, within the PTR scheme the ND texture component is depleted much faster than with the VFT scheme because grains with that orientation reach the required $50 \%$ twinned fraction in a few
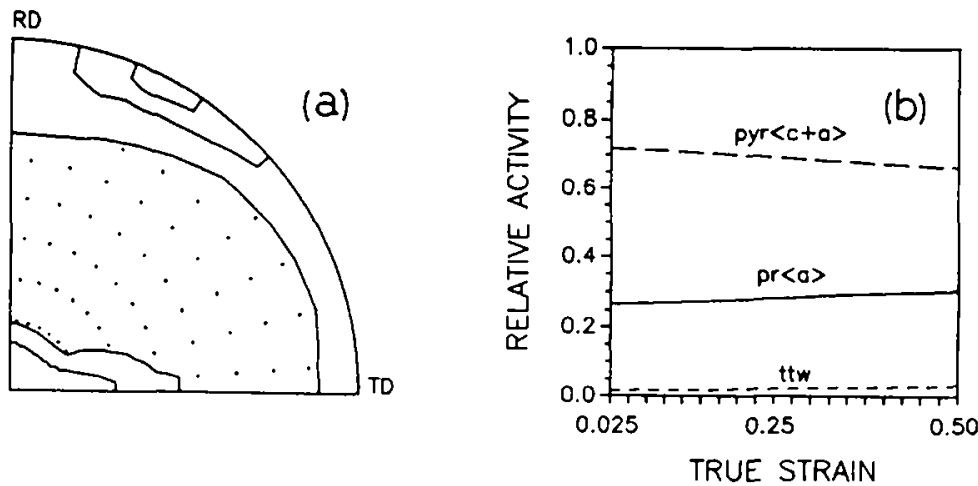

Fig. 4. Case $\tau^{\operatorname{pr}(a)}=1, \tau^{t t w}=2, \tau^{\operatorname{pyr}(e+a)}=4$ (see also caption to Fig. 3). (a) Basal pole figure obtained with the VFT scheme. (b) Contribution of each deformation mode to the total shear as a function of deformation for Fig. 4(a). 

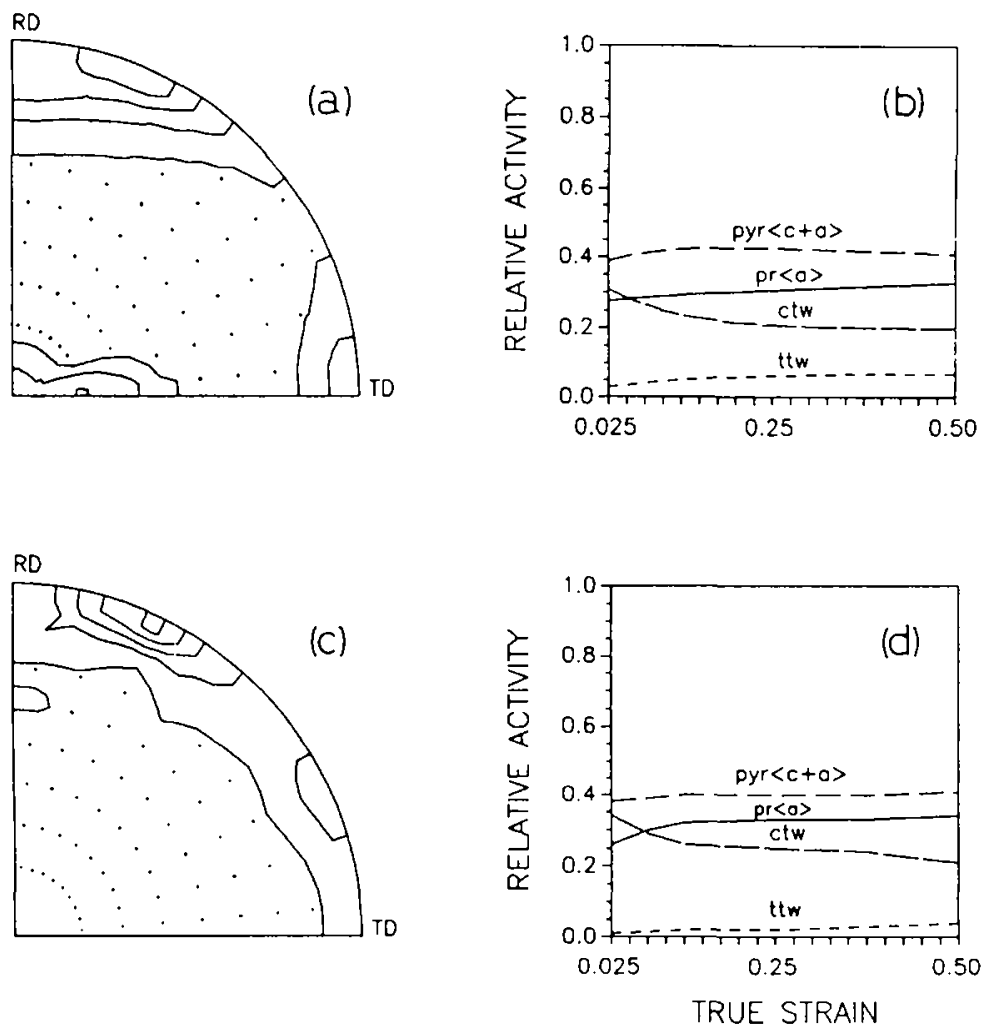

Fig. 5. Case $\tau^{p r\langle a\rangle}=1, \tau^{1 t w}=\tau^{c t w}=2$, $\tau^{p y r\langle c+a\rangle}=4$ (see also caption to Fig. 3). (a) Basal pole figure obtained with the VFT scheme. (b) Contribution of each deformation mode to the total shear for (a). (c) Basal pole figure obtained with the PTR scheme. (d) Contribution of each deformation mode to the total shear for (c).

steps and are reoriented as a whole, according to the PTR criterion.

When the CRSS's are shifted towards the topological domain 6 in Fig. $1\left(\tau^{\mathrm{pr}\langle a\rangle}=1, \tau^{\mathrm{tt \omega}}=\tau^{\mathrm{ct \omega}}=2\right.$, $\left.\tau^{\text {pyr }\langle c+a\rangle}>6\right),\langle c+a\rangle$ slip is deactivated and an even larger discrepancy between the predictions of the VFT and the PTR scheme is observed. The former gives a dominant component close to the ND [Fig. (6a)], a texture which closely resembles the one reported by Tenckhoff [22] for rolled zirconium. The PTR scheme gives a much less defined texture with no resemblance to the experimental ones [Fig. 6(c)]. This extreme case provides a crucial test for the twinning model because most of the deformation is accommodated by twinning, as can be inferred from the activity diagrams of Fig. 6(b) and (d). The discrepancy is due, as in the previous case, to the faster rate of reorientation by twinning associated with the PTR scheme. As a consequence, the RD and ND components are rapidly depleted, which also explains why the relative activity for the twinning systems is lower in Fig. 6(d) with respect to Fig. 6(b).

At this point we reach the partial conclusion that, when $\langle c+a\rangle$ pyramidal slip dominates, it leads to an unrealistic texture component. As a consequence, we either leave it out and accept that deformation is overwhelmingly accommodated by twinning or we introduce another slip mode and study how that affects the resulting textures. According to the discussion of Section 2 and the availability of systems in $\mathrm{Zr}$ alloys, deformation along the $c$-axis has to be accommodated either by $\langle c+a\rangle$ slip or by twinning, while prismatic slip is effective accommodating prismatic shears. As a consequence, the main role of the new system will be to take over from the other systems the shear activity in the basal plane. Slip in the basal plane in the $\langle a\rangle$ direction is known to be active only at high temperatures in zirconium $[6,23]$ and SalinasRodriguez has shown that accounting for it helps to explain high temperature texture development in zirconium alloys [24]. At low and intermediate temperatures it is likely that cross slip of $\langle a\rangle$ type in the pyramidal planes plays a role in accommoding basal shears. In what follows we are going to explore the consequences of incorporating this mode into our calculations.

The case when $\tau^{\text {pyr }\langle a\rangle} \simeq \tau^{\text {pyr }\langle c+a\rangle}$ [domain of low $\operatorname{pyr}\langle a\rangle$ in Fig. 1(b)] is not of interest because $\langle c+a\rangle$ slip dominates and the resulting texture is similar to the one shown in Fig. 3. Instead the combination $\tau^{\mathrm{pr}}=1, \tau^{\mathrm{pyr}\langle a\rangle}=2, \tau^{\mathrm{pys}\langle c+a\rangle}=4$ [domain 7 in Fig. 1(b)] favours the activity in the pyr $\langle a\rangle$ systems and leads to two stable components: the RD component associated with $\langle c+a\rangle$ slip and a partial fiber with a maximum tilted about $35^{\circ}$ from the ND towards the RD associated with $\langle a\rangle$ slip [see Fig. 7(a)]. The 

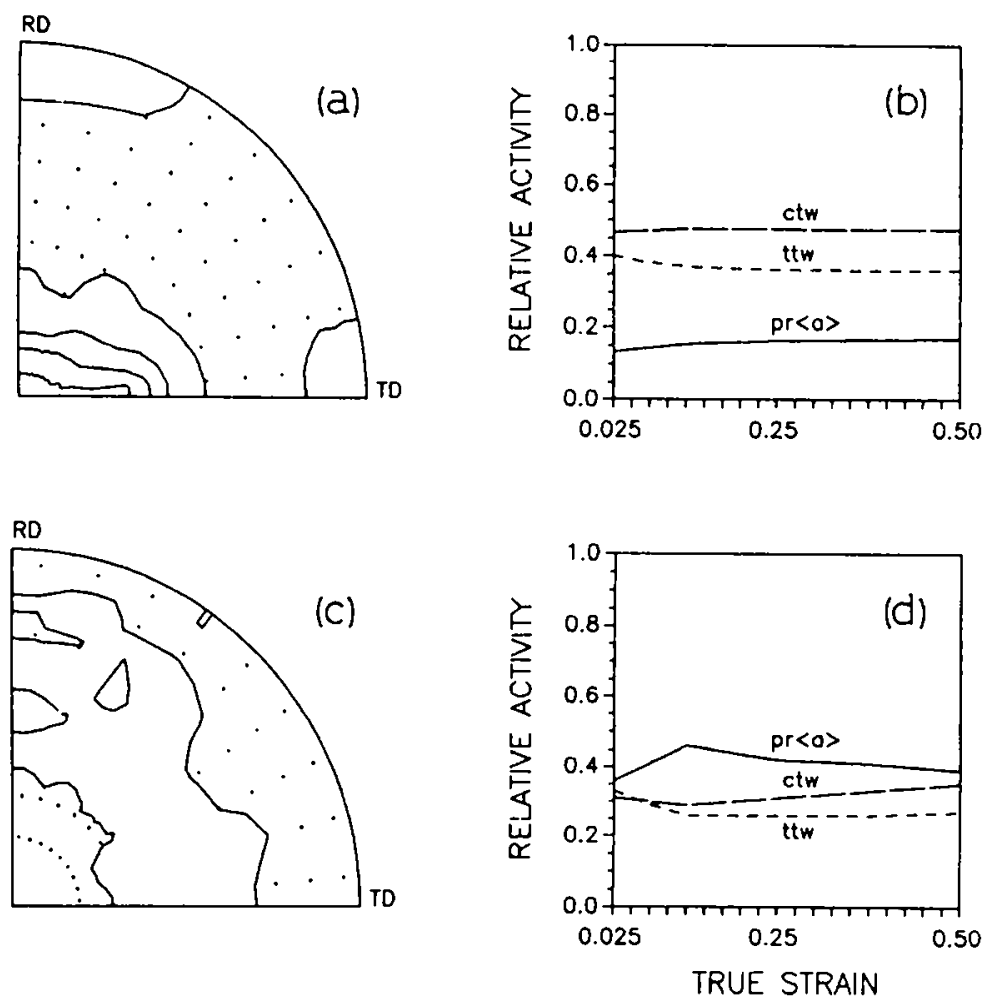

Fig. 6. Case $\tau^{\mathrm{pt}\langle a\rangle}=1, \tau^{\mathrm{tww}}=\tau^{\mathrm{ctw}}=2$ (sec also caption to Fig. 3). (a) Basal pole figure obtained with the VFT scheme. (b) Contribution of each deformation mode to the total shear for (a). (c) Basal pole figure obtained with the PTR scheme. (d) Contribution of each deformation mode to the total shear for (c).
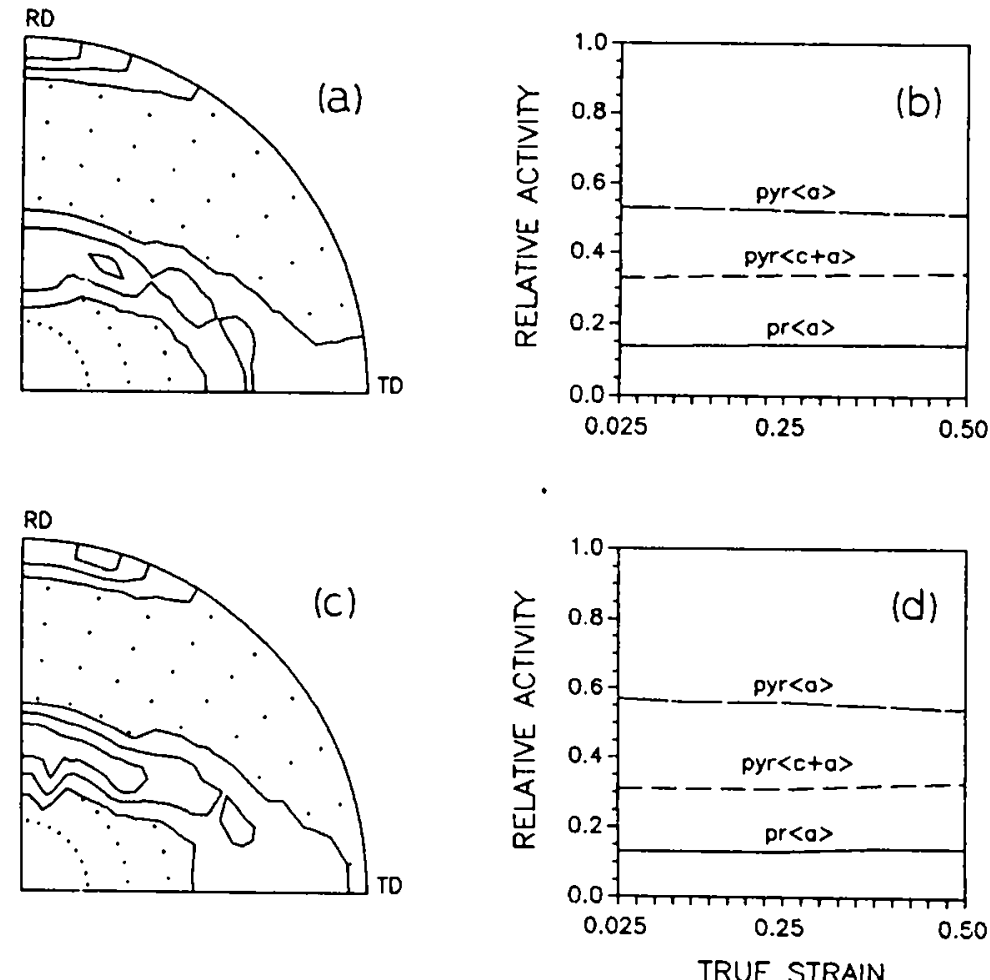

Fig. 7. Case $\tau^{p r\langle a\rangle}=1, \tau^{p y r(a)}=2, \tau^{p y r\langle c+a)}=4$ (see also caption to Fig. 3). (a) Basal pole figure obtained with the VFT scheme. (b) Contribution of each deformation mode to the total shear for (a). (c) Basal pole figure obtained with the PTR scheme. (d) Contribution of each deformation mode to the total shear for (c). 
present combination of active systems consisting only of slip modes, provides a test for the validity of the VFT method because the final texture does not depend on the twinning scheme being used. When the results derived with the VFT approach (fixed orientations and evolving volume fractions) are compared to the results of the classical approach (constant volume fractions and evolving orientations) it can be seen that both methods predict the same texture [Fig. 7(a) and (c)] and the same system activity [Fig. 7(b) and (d)].

The addition of tensile twins with $\tau^{\mathrm{ttw}}=2$ to the previous set of systems removes the RD component from the basal pole figure and leads to the fiber-type texture represented in Fig. 8(a). This texture has been measured by Thornburg and Piehler in titanium alloys [11], a material where $\langle a\rangle$ slip in pyramidal and basal planes is much well established. Ballinger [25] reports a similar texture in rolled Zircaloy-2, except that the fiber is closer to the ND and the maximum is at about 20 . Philippe et al. [8] calculate a texture similar to ours assuming the same active modes but the authors do not quote the values of CRSS's used in their calculation. The main contribution to deformation comes in this case from $\langle a\rangle$-pyramidal slip and the contribution of twinning decreases gradually as deformation proceeds [see Fig. 8(b)]. The fact that basically the same texture and the same system activity is predicted with the PTR scheme [see Figs 8(c) and (d)] indicates that the latter
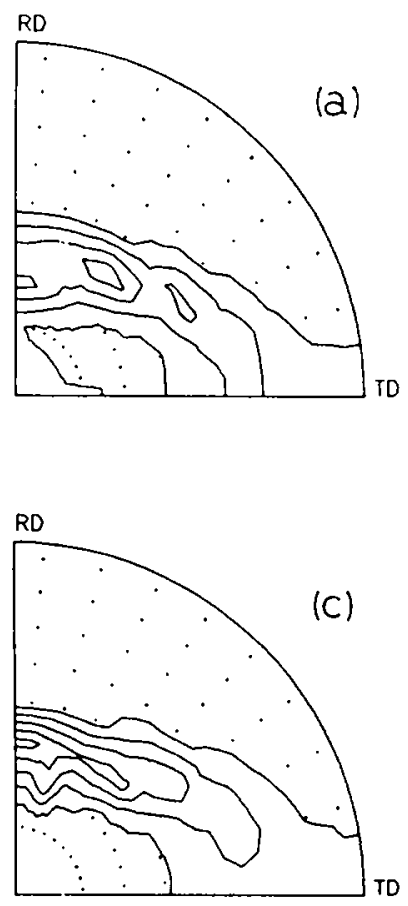

is applicable as far as twinning is only moderately active.

The case when compressive twins with $\tau^{\mathrm{ctw}}=2$ are added to the previous set of systems corresponds to domain 9 of Fig. 1(b). The compressive twins replace the pyr $\langle c+a\rangle$ systems in accommodating compression along the $c$-axis, as can be seen from a comparison of Figs $8(\mathrm{~b})$ and $9(\mathrm{~b})$ giving the mode activity. This has the effect of spreading the texture, which now exhibits two weak components in the TD and the ND [see Fig. 9(a)]. Here again the texture predicted with the VFT scheme differs from the one predicted with the PTR scheme [Fig. 9(c)] although Fig. 9(b) and (d) indicate that the associated activities are similar. Once again, the discrepancy is due to the fact that when twinning activity is high the results depend critically upon the twinning scheme being used.

As a conclusion to this section: the calculated texture shown in Fig. 6(a) is exclusively due to twinning reorientation and is in good agreement with experimental pole figures of rolled zirconium alloys. Also Fig. 8(a) displays a texture which is sometimes observed. The difference between both cases is the unusual activity in the pyramidal $\langle a\rangle$ sytems required by the latter, with twinning being practically inactive.

\subsection{Tension and compression textures}

Although a detailed analysis of tensile and compressive textures in hexagonal metals is presented
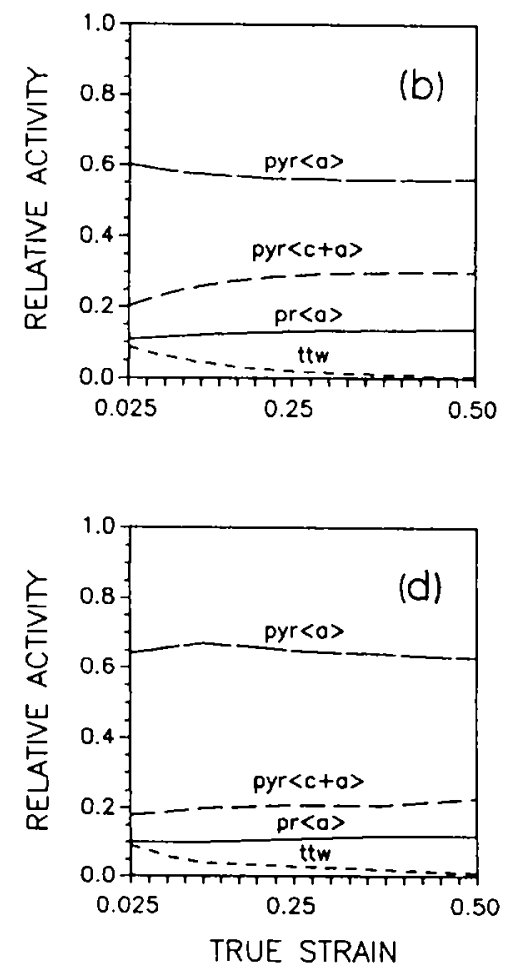

Fig. 8. Case $\tau^{\mathrm{pr}\langle a\rangle}=1, \tau^{\mathrm{pyr}\langle a\rangle}=\tau^{\mathrm{ttw}}=2, \tau^{\mathrm{pyr}\langle c+a\rangle}=4$ (see also caption to Fig. 3). (a) Basal pole figure obtained with the VFT scheme. (b) Contribution of each deformation mode to the total shear for (a). (c) Basal pole figure obtained with the PTR scheme. (d) Contribution of each deformation mode to the total shear for (c). 

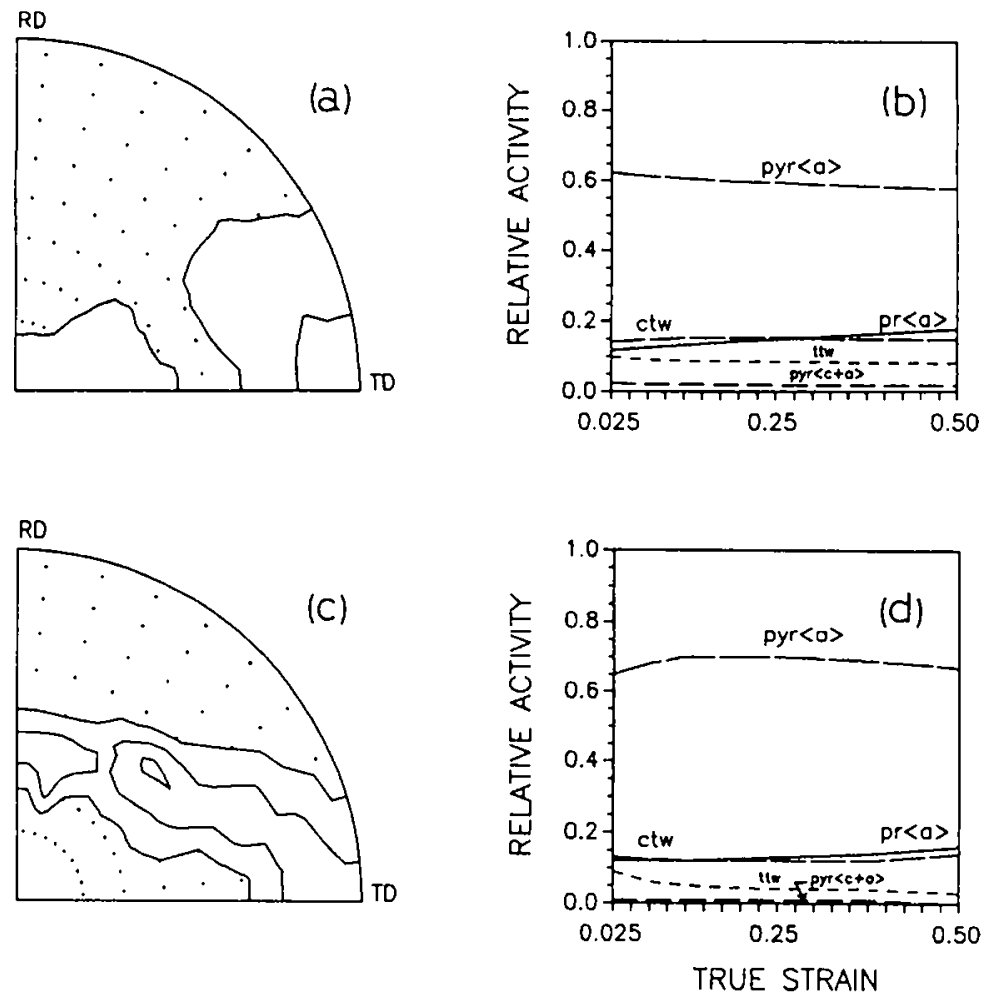

Fig. 9. Case $\tau^{p r\langle a\rangle}=1, \tau^{\mathrm{pyr}\langle a\rangle}=\tau^{\mathrm{tw} w}=\tau^{\mathrm{ctw}}=2, \tau^{\mathrm{pyr}\langle c+a\rangle}=4$ (see caption to Fig. 3). (a) Basal pole figure obtained with the VFT scheme. (b) Contribution of each deformation mode to the total shear for (a). (c) Basal pole figure obtained with the PTR scheme. (d) Contribution of each deformation mode to the total shear for (c)

elsewhere $[5,26]$, we would like to explore briefly in this section the combinations of active systems which lead to reasonable rolling textures and use them to predict tension and compression textures.

In what follows, only the VFT scheme will be used to simulate axisymetric deformation in tension and compression up to $50 \%$ true strain and starting from a non-textured polycrystal. Axisymmetry makes it unnecessary to plot complete basal pole figures because the isointensity lines consist in circles concentric with the axial direction. As a consequence, calculated basal pole intensities are plotted in Fig. 10 as a function of the tilting of the basal poles with respect to the tensile or compressive axis.

The simulations are performed for combinations of systems corresponding to Figs $6(\mathrm{pr}\langle a\rangle$, ttw, ctw) and $8(\operatorname{pr}\langle a\rangle, \operatorname{pyr}\langle a\rangle, \operatorname{pyr}\langle c+a\rangle$, ttw) and the results are plotted in Fig. 10. The same as for rolling, we find that the final basal pole distribution shows distinctive characteristics, depending on the combination of active systems chosen. The activation of 〈a prismatic, tensile twins and compressive twins gives a distribution of basal poles perpendicular to the tensile axis in tension and along the compressive axis in compression [Fig. 10(a)]. This type of texture, associated with high twinning activity, has been reported in Zircaloy-2 by MacEwen et al. [27] and is explored in detail in Ref. [26]. As for the other combination, characterized by high slip activity and the absence of compressive twins, it predicts a maximum $65^{\circ}$ away from the tensile axis and $35^{\circ}$ away from the compressive axis respectively [Fig. 10(b)]. A compression texture with similar characteristics has been measured by Salinas-Rodriguez [28] in $\mathrm{Zr}-2.5 \% \mathrm{Nb}$ of small grain size.

\section{CONCLUSIONS}

Polycrystal plasticity and texture development are complex to simulate in materials of hexagonal structure because of the intrinsic plastic anisotropy of the constituent grains. Typically, the deformation mechanisms encountered in these materials consist in several slip and twinning modes for which the associated critical stresses depend on previous deformation history, temperature, alloy content and grain size, and which are usually not accessible to direct measurement. In this respect the comparison between predicted and measured textures provides an indirect way of inferring information about the active deformation modes. Furthermore, when twinning is active the results depend critically on the approximation used to account for twinning reorientation during the simulation and a reliable model is required in this respect. We concentrate here on the treatment of the 


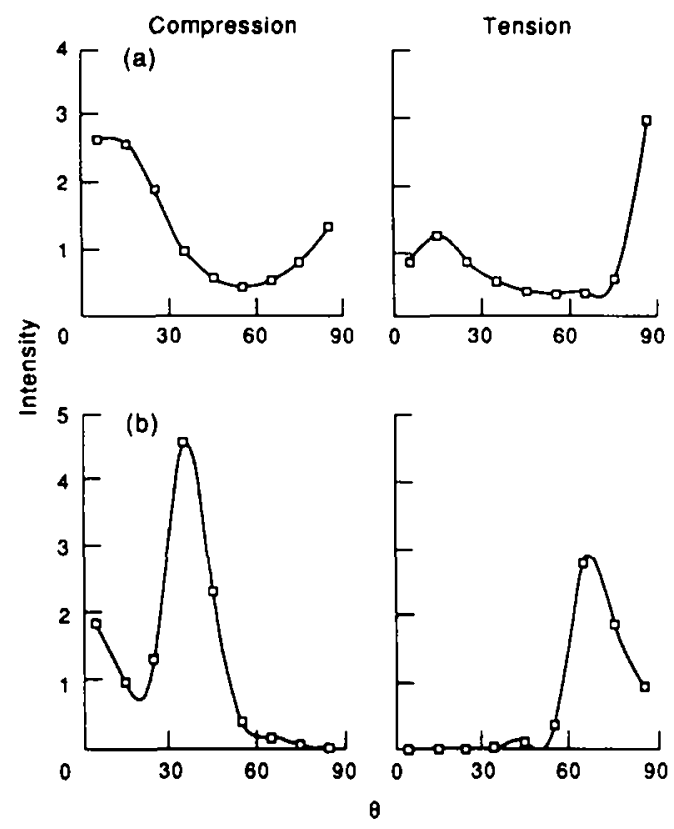

Fig. 10. Basal pole intensities obtained with the VFT scheme under FC conditions after 0.5 true strain in axisymmetric compression and tension. $\theta$ is the tilting of the $c$-axis with respect to the tensile and the compressive axis. (a) Case $\tau^{p \nmid\langle a\rangle}=1, \quad \tau^{\mathrm{ttw}}=\tau^{\mathrm{ctw}}=2$. (b) Case $\tau^{\mathrm{pr}\langle a\rangle}=1$, $\tau^{\text {pyr }\langle a\rangle}=\tau^{t \mathrm{tw}}=2, \tau^{\text {pyr }\langle c+a\rangle}=4$.

twinned volume fractions: no attempt is made to account for other effects such as hardening, the interaction between grains and matrix, or the local strain accommodation caused by the activation of twinning. Although each of these aspects is relevant to a complete understanding of the mechanical behavior of the material, we consider that they have to be analyzed separately in order to elucidate their respective importance.

A Monte-Carlo approach was judged to be impractical because of the prohibitively large number of initial orientations that its application requires and, in this work, we test two different novel methods for dealing with twinning reorientation. The Predominant Twinning Reorientation (PTR) scheme is along the classical lines: grains are reoriented as deformation proceeds without modifying their associated volume fraction and the total number of orientations representing the polycrystal is kept constant. A grain is completely reoriented by twinning acording to a criterion based on the twinning history of the grain. The results obtained in this work indicate that this approach is valid when twinning is not the dominant deformation mechanism. Otherwise, reorienting the whole grain when $50 \%$ (or some other fraction) of the grain has reoriented on the predominant twinning system leads to unrealistic textures. For such cases, splitting the grain when a non-negligible fraction of it has twinned in a given system will probably result in improved textures without leading to a prohibitively large number of orientations. We are presently exploring the consequences of such an approach [5]. However, our experience indicates that minor improvement in the predicted textures is to be expected along this line.

The VFT scheme is a novel formulation based on a different modelling philosophy, which consists in working with a fixed set of orientations while allowing for the associated volume fractions to evolve with deformation. This approach provides not only a way for keeping track exactly of the twinned fractions but it also allows to tackle grain reorientation due to slip. Besides, the scheme is suited for modelling recrystallization and phase transformation textures. According to the results derived in this work the VFT approach performs better than classical approaches when twinning makes an important contribution to deformation and makes no difference when deformation is accommodated by slip only. A minor disadvantage is that a larger (but still tractable) number of orientations is required, although this disadvantage is compensated by a substantial increase in the processing speed when equal strain increments are imposed.

Concerning the dependence of the textures with the assumed active systems and their CRSS's, we present in this work a systematics for dealing with the problem, based on a previous topological analysis of the Single Crystal Yield Surface and the identification of domains of CRSS's leading to the same system activity (and so to the same final texture). By reducing the analysis to a few sets of CRSS's and their associated textures, this method narrows considerably the cases to be considered and provides a better understanding of the relation between active systems and deformation textures.

We implement the method to the specific case of zirconium alloys and the active deformation modes that they exhibit. We show that whenever $\langle c+a\rangle$ pyramidal slip dominates it leads to texture components which are not experimentally observed. We also show that it is possible to explain the experimental textures assuming that prism slip and twinning are the only deformation mechanisms [Figs $6(a)$ and 10(a)]. However, the addition of $\langle a\rangle$ pyramidal slip may also lead to observed texture [Figs 8(a) and 10(b)]. In agreement with experimental observation, it is unlikely that a unique combination of systems exists that explains all deformation textures, but different $\mathrm{Zr}$ alloys will require different active modes for that purpose.

No attempt is made in this work to account for hardening and as a matter of fact the CRSS ratios between the different modes are kept constant during deformation. This may be a strong assumption but there is no direct experimental evidence that permits to infer the way in which different modes interact as a function of deformation. It is possible, however, to infer qualitative information about hardening as follows: hardening describes the evolution of the CRSS's with deformation and such evolution is represented by a trajectory across the topological map of 
the SCYS. The textures calculated in Section 4 (and represented in Figs 3-9) are invariant as far as the combination of CRSS's remains within the corresponding topological domain of Fig. 1. As a consequence, only when the representative point crosses a topological boundary will the hardening affect the reorientation tendency and therefore the texture evolution. As an example of such evolution, consider the case where deformation starts at domain 4 in Fig. 1 and assume that hardening originates a transition to domain 6 going through domain 5 . At the beginning compressive twins are not active and a texture like the one in Fig. 4 tends to form, with a RD component due to $\operatorname{pyr}\langle c+a\rangle$ activity and a ND component due to tensile twinning. A faster hardening of the pyr $\langle c+a\rangle$ systems shifts the CRSS's to domain 5 , and compressive twins take over part of the $\langle c+a\rangle$ activity, helping tensile twins to deplete the RD component and to reinforce the component along the ND. Further hardening of the pyr $\langle c+a\rangle$ deactivates those systems and brings the CRSS's to domain 6 , where the texture component is stable under twinning activity.

We are aware of the fact that a Fully Constrained or a Relaxed Constraints approach may be too restrictive for treating this type of material and we are simultaneously exploring the consequences of using self-consistent formulations which allow for different strain increments from grain to grain. We expect that certain texture components which are not stable under FC or RC conditions will be stable under self-consistent conditions. We also recognize that within the VFT scheme, hardening is not dealt with in a deterministic way, but somewhat statistically because material is transferred between cells and accumulated to the existing orientation disregarding its previous deformation history. Nevertheless, a sound twinning reorientation scheme and a topological characterization of the deformation modes are prerequisites if a polycrystal plasticity model is to be successful and we believe that this work establishes a basis for the fulfillment of those conditions.

Acknowledgements - The authors wish to thank Dr Armando Salinas-Rodriguez for valuable comments on the manuscript and for providing experimental information prior to publication.

\section{REFERENCES}

1. C. N. Tomé and U. F. Kocks, Acta metall. 33, 603 (1985).

2. P. Lequeu, P. Gilormini, F. Montheillet, B. Bacroix and J. J. Jonas, Acta metall. 35, 439 (1987).

3. G. Y. Chin and W. L. Mammel, Metall. Trans. 1, 357 (1970).

4. T. Takeshita, C. N. Tomé, H.-R. Wenk and U. F. Kocks, J. Geophys. Res. 92, 12917 (1987).

5. U. F. Kocks and C. N. Tomé, submitted for publication.

6. E. Tenckhoff, in Zirconium in Nuclear Applications, ASTM STP 551, p. 179 (1974).

7. M. J. Philippe, F. Wagner and C. Esling, Proc. Eighth Int. Conf. on Texture of Materials ICOTOM -8 (edited by J. S. Kallend and G. Gottstein), p. 837. T.M.S. Warrendale, $\mathrm{Pa}$ (1988).

8. M. J. Philippe, C. Esling and B. Hocheid, Text. Microstruct. 7, 265 (1988).

9. G. Y. Chin and W. L. Mammel, Trans. metall. Soc. AIME 245, 1211 (1969).

10. J. F. W. Bishop and R. Hill, Phil. Mag. 42, 414 (1951).

11. D. R. Thornburg and H. R. Piehler, in Titanium Science and Technology (edited by R. I. Jaffee and H. M. Burte), Vol. 2, p. 1187. Plenum Press, New York (1973).

12. D. R. Thornburg and H. R. Piehler, Metall. Trans. A 6, 1511 (1975).

13. P. Van Houtte, Acta metall. 26, 591 (1978).

14. H.-R. Wenk, T. Takeshita, R. Jeanloz and G. C. Johnson, Geophys. Res. Lett. 15, 76 (1988).

15. S. K. Schiferl, J. appl. Phys. 66, 2637 (I989).

16. C. N. Tomé, G. R. Canova, S. Ahzi and A. Molinari, same as Ref. [7], p. 395.

17. Th. Steinkopff and S. Matthies, same as Ref. [7], p. 49.

18. C. Esling, H. J. Bunge and J. Muller, same as Ref. [7], p. 295.

19. H. Klein, C. Esling and H. J. Bunge, same as Ref. [7], p. 307.

20. G. R. Canova and U. F. Kocks, Proc. Seventh Int. Conf. on Texture of Materials ICOTOM -7 (edited by C. M. Brakman, P. Jongenburger and E. J. Mittemeijer), p. 573. Noordwijkerhout, The Netherlands (1984).

21. R. A. Lebensohn, Master thesis, Universidad Nacional de Rosario (1989).

22. E. Tenckhoff, Metall. Trans. 9A, 1401 (1978).

23. A. Akhtar, Acta metall. 21, 1 (1973).

24. A. Salinas-Rodriguez, Ph.D. Thesis, McGill Univ., Canada (1988).

25. R. G. Ballinger, The Anisotropic Mechanical Behavior of Zircaloy-2. Garland, New York (1979).

26. R. A. Lebensohn and C. N. Tomé, Proc. Ninth Int. Conf. on Texture of Materials (ICOTOM-9), Avignon, France (1990). To be published.

27. S. R. MacEwen, N. Christodoulou, C. N. Tomé, J. Jackman, T. M. Holden, J. Faber Jr and R. L. Hitterman, same as Ref. [7], p. 825.

28. A. Salinas-Rodríguez, private communication. 